\title{
TAGUNG
}

\section{Die gemeinschaftliche Außenwirtschaftspolitik im Konstitutionalisierungsprozess}

\author{
Philipp Jehle*
}

Eine kritische Reflexion des gegenwärtigen Zustandes der Europäischen Gemeinschaft hierzu gibt die jüngste Phase des Ratifizierungsprozesses angesichts der gescheiterten Verfassungsreferenden in Frankreich und den Niederlanden fordernden Anlass. Die gemeinsame Außenwirtschaftspolitik kann hierbei als Bezugspunkt der Betrachtungen von hervorragendem Wert sein. Die Tagung mit dem Titel „Die Außenwirtschaftspolitik der Europäischen Union nach dem Verfassungsvertrag “, welche am 7. und 8. Juli 2005 in München stattfand, hat sich dieser aktuellen Aufgabe angenommen.

Zum Auftakt der Veranstaltung führte Christoph Herrmann in das Tagungsthema ein. Er wies auf das ungewisse Schicksal des Vertrags über eine Verfassung für Europa (VVE) hin. Diese derzeit rechtskraftlose Regulierungsleistung berge für den Bereich der AuBenwirtschaftspolitik und dessen Analyse jedenfalls vielseitiges Diskussionspotenzial. Insbesondere die Einordnung der Gemeinsamen Handelspolitik (GHP) in den Bezugsrahmen des allgemeinen auswärtigen Handelns der Gemeinschaft, die Untersuchung der Kompetenzverlagerungen und die Wechselwirkungen mit dem Recht der Welthandelsorganisation seien hierbei von vorrangiger Wichtigkeit. Denn selbst bei einem endgültigen Scheitern des Ratifizierungsprozesses werde sich die Gemeinschaft der im Verfassungsvertrag gefundenen Lösungsoptionen voraussichtlich auf anderen Pfaden der Rezeption zumindest partiell zu bedienen versuchen.

\section{Die Außenwirtschaftspolitik der EU nach dem Verfassungsvertrag}

Wissenschaftliches Symposium des Arbeitskreis Europäische Integration (AEI) und des Lehrstuhls für Öffentliches Recht und Europarecht und des Instituts für Internationales Recht, Abteilung Völkerrecht, der Ludwig-Maximilians-Universität München, in Zusammenarbeit mit der IHK für München und Oberbayern München, 7./8. Juli 2005

\section{Eröffnung und Begrüßung}

Prof. Dr. Rudolf STREINZ, Ludwig-Maximilians-Universität München

Dipl.-Volksw. Helgo ALBERTS, IHK für München und Oberbayern

Prof. Dr. Wulfdiether ZIPPEL, AEI e.V., Hochschule für Politik München

Einführung in das Tagungsthema

Dr. Christoph HERRMANN, LL.M., Ludwig-Maximilians-Universität München

Panel I: Die Gemeinsame Handelspolitik als integrierter Bestandteil des auswärtigen Handelns der EU

Ziele der Gemeinsamen Handelspolitik Prof. Dr. Christoph VEDDER, Universität Augsburg

Das institutionelle Gleichgewicht, insbesondere die Rolle des Parlaments

Prof. Dr. Markus KRAJEWSKI, Universität Potsdam

Die Gemeinsame Handelspolitik als Dienerin der GASP?

Prof. Dr. Jörg MONAR, University of Sus$\operatorname{sex}$

\footnotetext{
* Philipp Jehle, Wissenschaftlicher Angestellter, Institut für deutsches und europäisches Gesellschafts- und Wirtschaftsrecht, Universität Heidelberg.
} 
Gemeinsame Handelspolitik und auswärtiges Handeln

Zunächst überprüfte Christoph Vedder die These, die Gemeinsame Handelspolitik stelle sich nach den Regelungen des Verfassungsvertrages als ein integrierter Bestandteil des allgemeinen auswärtigen Handelns der Union dar. Hierzu unterzog Vedder die Zielkataloge beider Regelungskomplexe in den Artt. III292 VVE und III-315 VVE einer Analyse. Zwar handele es sich hinsichtlich des Umfangs und des Standorts um innovative Regelungen, in der Sache brächten sie indes nichts Neues. Dies belege beispielsweise ein Vergleich mit Art. 11 EUV und mit anderen Normen der derzeit gültigen Verträge. Die explizite Aufnahme interner Leitprinzipien in den Vorgabenkatalog des Art. III-292 VVE verstärke bloß deren bereits eingeläutete Transformation in ein Konzept integraler Weltordnungspolitik. Art. III-315 Abs. 1 VVE bilde die faktisch bereits bestehende Aufgabenwahrnehmung in der Welthandelsorganisation kongruent $\mathrm{ab}$, indem er die Erweiterung der Gemeinschaftskompetenzen um die Bereiche des Dienstleistungshandels, der handelsbezogenen Aspekte des geistigen Eigentums und der ausländischen Direktinvestitionen vorsehe. Zwar erlaube die Norm neben der Verwirklichung explizit handelsspezifischer Interessen auch die Verfolgung ordnungspolitischer Ziele wie beispielsweise der Friedens- und Sicherheitspolitik oder aber der Entwicklungs-, Umwelt- und Menschenrechtspolitik durch Mittel der Handelspolitik. Hierin sei eine verstärkte Hinwendung der Gemeinschaft $\mathrm{zu}$ einer offenen, wertebezogenen Politik zwar niedergelegt. Eine Instrumentalisierung der Gemeinsamen Handelspolitik vermochte Vedder indes nicht $\mathrm{zu}$ erkennen. Vom engen systematischen Kontext beider Regelungskomplexe allein lasse sich nicht auf eine Unterordnung der Handelsaspekte unter ordnungspolitischen Prämissen schließen.

Im Folgenden beschäftigte sich Markus Krajewski mit den Auswirkungen der Änderungen durch den Verfassungsvertrag auf das
Panel II: Die ,neuen“6 Bereiche der Gemeinsamen Handelspolitik und ihre fortbestehenden Besonderheiten

Handel mit Dienstleistungen

RA Dr. Christian PITSCHAS, LL.M., MSBH Rechtsanwälte, Genf

Handelsaspekte und sonstige Aspekte geistigen Eigentums sowie Investitionen

RA Dr. Till MÜLLER-IBOLD, Cleary, Gottlieb, Stehen \& Hamilton, Brüssel

Panel III: Rechtsinstrumente und Rechtsschutz im Rahmen der Gemeinsamen Handelspolitik

Die zukünftigen Rechtsinstrumente der Gemeinsamen Handelspolitik

Dr. Christoph HERRMANN, LL.M., Ludwig-Maximilians-Universität München

Rechtsschutz, gegen hoheitliche Eingriffe in die Außenwirtschaftsfreiheit

Prof. Dr. Martin NETTESHEIM, Universität Tübingen

Panel IV: Die Reform der Gemeinsamen Handelspolitik und das WTO-Recht: Konsequenzen und Wechselwirkungen

Das Ende der parallelen Mitgliedschaft von EU und Mitgliedstaaten in der WTO?

Prof. Dr. Christian TIETJE, Universität Halle-Wittenberg

WTO-konforme Auslegung und Staatshaftung: Neue Perspektiven nach dem Verfassungsvertrag?

PD Dr. Wolfgang WEISS, Universität Bayreuth

Abschlusspanel: „Harnessing Globalisation" - Ausblick und Perspektiven

Moderation und Abschlussstatement:

RA Prof. Dr. Horst G. KRENZLER, Institut für Internationales Recht, Ludwig-Maximilians-Universität München

Panelstatements:

Prof. Dr. Meinhard HILF, Bucerius Law School, Hamburg

Dr. Karl-Ernst BRAUNER, Ministerialdirektor, BMWA, Berlin

Dr. Marc BEISE, Süddeutsche Zeitung, München

RA Dr. Till MÜLLER-IBOLD, Cleary, Gottlieb, Stehen \& Hamilton, Brüssel 
Kompetenzgefüge der Gemeinschaftsorgane. Während Art. 300 Abs. 3 EGV in der Regel nur eine Anhörungspflicht des Europäischen Parlaments bestimme, sehe der Verfassungsvertrag in Art. III-325 Abs. 6 VVE für internationale Übereinkünfte eine Ausweitung des Zustimmungserfordernisses vor. Diese leiste einer Parlamentarisierung des Außenhandelssektors Vorschub, wobei Tendenzen hin zu einem „quasipräsidialen Regierungssystem“ im Handelsbereich ähnlich dem US-amerikanischen zu erkennen seien. Es folgten Ausführungen zum vorgesehenen Amt eines EUAußenministers, wobei nach Meinung Krajewskis schon die Errichtung dieser Position durch ihre bipolare Einbindung in Rat und Kommission eine Schwächung der Rolle der Kommission mit sich bringen werde, woraus eine verstärkte Politisierung des Außenhandelsregimes resultieren könne. Ferner bestehe in der EU-Bevölkerung ein positives Stimmungsbild in Bezug auf die Außenhandelspolitik. Insofern sei eine Einführung der GHPRegelungen auch abseits des Weges der Ratifikation unter Legitimationsaspekten durchaus wünschenswert. Diesbezüglich wies Krajewski drei Wege auf: das Vertragsänderungsverfahren nach Art. $48 \mathrm{EUV}$, die Implementierung durch interinstitutionelle Vereinbarungen sowie die verfassungsnahe Interpretation von EGV und EUV. Krajewski kam zu dem Schluss, dass eine Parlamentarisierung der Außenhandelsbeziehungen auch ohne Ratifikation des Verfassungsvertrages durch eine parlamentsfreundliche Auslegung der bestehenden Verträge möglich und erstrebenswert sei. Hingegen bedürfe eine weitere Politisierung dieses Sektors der Ratifikation selbst.

Jörg Monar wandte sich der Fragestellung zu, ob die Gemeinsame Handelspolitik als Dienerin der Gemeinsamen Außen- und Sicherheitspolitik (GASP) zu betrachten sei. Monar erkannte ein Primat der GASP, welches sich bereits den Zielsetzungsoptionen aus Art. III315 VVE entnehmen lasse. Auch führe die Doppelfunktion des Außenministers zumindest potenziell zu einer verstärkten Bedin- gung der Handelspolitik durch handelsfremde Motivationen. Auch könne durch Art. III-325 Abs. 3 VVE bezüglich der Frage, ob eine Maßnahme hauptsächlich Handelsaspekten oder aber der Sicherheitspolitik diene, dem Außenminister eine gewisse Einschätzungsprärogative zuerkannt sein. Da die sektorale Zuordnung einer Maßnahme indes erhebliche Auswirkung auf die Kontrollzuständigkeit des EuGH habe, müsse die etwaige Ausübung dieses Vorrechtes sorgsam verfolgt werden. Die Außenhandelspolitik habe sich im Laufe der Jahrzehnte als Erfolgsgeschichte und echter Wohlstandsförderer für die Bürger der Gemeinschaft bewährt. Eine Instrumentalisierung sei daher insbesondere unter dem Gesichtspunkt der „output legitimacy“ nicht unkritisch.

\section{Erweiterte Kompetenzen - Dienstleistungen, geistiges Eigentum und Direktinvestitionen}

Christian Pitschas untersuchte die Regelungen des Verfassungsvertrages bezüglich des Handels mit Dienstleistungen. Hierzu skizzierte er die Geschichte der Kompetenzverteilung nach dem Grundsatz der geteilten $\mathrm{Zu}-$ ständigkeit zwischen der exklusiven Gemeinschaft und den Mitgliedstaaten. Nachdem durch den Konvent diverse Reformansätze diskutiert worden waren, sehe nun Art. III-315 Abs. 1 VVE die Übertragung der ausschließlichen Kompetenz für den Bereich des Handels mit Dienstleitungen auf die Gemeinschaft vor. Auch Regelungen zum Dienstleistungsbereich, die sich auf den freien Personenverkehr oder sonstige Grundfreiheiten auswirkten, wären im Gegensatz zur bisherigen Rechtslage hiernach von der exklusiven Gemeinschaftskompetenz erfasst. Diese Kompetenz erstrecke sich allerdings nicht auf Maßnahmen, mit denen eine mittelbare Harmonisierung mitgliedstaatlicher Rechtsvorschriften einhergehen würde (Art. III-315 Abs. 6 VVE). Diese Maßgabe könne zum Beispiel im Rahmen von Abkommen über die gegenseitige Anerkennung beruflicher Qualifikationen Relevanz erlangen. Für Abstimmungen im Rat hinsichtlich der Ausarbeitung 
eines internationalen Abkommens genüge auch im Bereich des Dienstleistungshandels grundsätzlich ein qualifiziertes Mehrheitsvotum. Ein Einstimmigkeitserfordernis sei daher die Ausnahme, welche insbesondere in den sensibeln Bereichen von audiovisuellen und kulturellen Dienstleistungen sowie Dienstleistungen im Bildungs-, Gesundheits- und Sozialwesen aufrechterhalten bleibe (Art. III-315 Abs. 4 VVE). Zudem wies Pitschas darauf hin, dass die deutsche Fassung nach dem Wortlaut dann Einstimmigkeit erfordere, wenn die kulturelle und sprachliche Vielfalt „,in der Union“ beeinträchtigt werde, was bereits dann anzunehmen sein könnte, wenn ein Mitgliedstaat eine Beeinträchtigung in seinem Hoheitsgebiet erkenne. Die französische und die englische Fassung indes setzten voraus, dass die entsprechende Vielfalt der Union beeinträchtigt sei. Hier bestehe Klärungsbedarf.

Till Müller-Ibold befasste sich mit handelsbezogenen und sonstigen Aspekten des geistigen Eigentums und von Investitionen. Für die bisherige Gemeinschaftsrechtslage zeichnete er ein Bild des uneinheitlichen Schutzes geistigen Eigentums. Mit dem Vertrag von Nizza seien aber zumindest große Teile der handelsbezogenen Aspekte geistigen Eigentums in Gemeinschaftskompetenz überführt worden. Hier habe auf der Ebene des Rechtsschutzes und des Verfahrensrechtes auch eine innergemeinschaftliche Rechtsangleichung insbesondere durch die Richtlinie 2004/48 stattgefunden. Für den Sektor der handelsbezogenen Aspekte von Investition fänden sich im Nizza-Vertrag keinerlei Regelungen, dennoch hätte die Kommission gewisse, bisher aber ungenutzte Gemeinschaftskompetenzen aus den Befugnissen bezüglich des freien Zahlungsverkehrs abgeleitet. Die Mitgliedstaaten hingegen hätten durch eine große Zahl bilateraler Investitionsschutzabkommen massive Regelungsaktivität entwickelt. Der Verfassungsvertrag sehe nun eine Überführung der Regelungsmaterien handelsbezogener Aspekte geistigen Eigentums und - unter Durchbrechung des kompetenzrechtlichen Parallelitätsgrundsatzes - des Bereichs der ausländischen Direktinvestitionen in ausschließliche Gemeinschaftskompetenz vor (Art. III-315 Abs. 1 VVE). Dass allerdings die Regulierung der in der Praxis ebenfalls relevanten kurzzeitigen Portfolio-Investitionen hiervon nicht erfasst sei, könne bedauert werden.

\section{Wirkmittel und Rechtsschutz im Bereich der Außenhandelspolitik}

Christoph Herrmann erläuterte in Vertretung des erkrankten Wolfgang Müller die Rechtsinstrumente im Bereich der Gemeinsamen Handelspolitik ausgehend von der traditionellen Dominanz der Rechtsverordnung. Der Verfassungsvertrag sehe nun eine Reduzierung und Revision der bisher 15 anerkannten Arten von Rechtsakten auf sechs Instrumente vor (Art. I33 Abs. 1 VVE). Wesentlichen Regelungen seien durch Instrumente mit Gesetzescharakter zu treffen, eine strenge Normenhierarchie mit Anleihen aus dem Staatsrecht werde installiert. Nach Art. III-315 Abs. 2 VVE sei der Rahmen der Gemeinsamen Handelspolitik durch ein Europäisches Gesetz festzulegen. Auf Grund der Ausgestaltung des gemeinschaftsrechtlichen Gesetzgebungsverfahrens unterläge diese Rahmenfestlegung dann der parlamentarischen Kontrolle durch das Europaparlament. Der Vollzug der Regelung habe gerade für den Bereich von Antidumping-, Antibeihilfen- und sonstigen Schutzmaßnahmen durch die Europäischen Durchführungsverordnung gemäß Art. I-37 Abs. 4 VVE und nicht anhand der Delegierten Europäischen Verordnung nach Art. I-36 VVE zu erfolgen, da hier ausnahmsweise ein gemeinschaftseigener Vollzug erforderlich sei.

Mit dem Schutz des Bürgers vor hoheitlichem Eingriff in die Außenhandelsfreiheit befasste sich anschließend Martin Nettesheim. Nach der bisherigen Rechtslage und Rechtsprechung bestünde eine Klagebefugnis unter abnehmender Relevanz des Typus des jeweiligen Rechtsaktes dann, wenn eine unmittelbare und individuelle Betroffenheit gegeben sei. Eine zusätzliche Eröffnung von Klagemög- 
lichkeiten habe die europäische Rechtsprechung nicht gewährt und diese Aufgabe dem Verfassungskonvent überlassen. Dieser habe nun in Art. III-365 Abs. 4 VVE eine neue Regelung getroffen, durch welche die Art der Maßnahme auf Grund der hieran anknüpfenden unterschiedlichen Zulässigkeitsvoraussetzungen wieder verstärkte Relevanz erlangen könne. Insbesondere die Einordnung des Beschlusses bereite hier Schwierigkeiten. Rechtsschutzlücken seien im Bereich der Maßnahmen mit Rechtswirkung von Nebenorganen, Einrichtungen und sonstigen Stellen der Union geschlossen worden (Art. III-365 VVE). Eine Zuständigkeit der Rechtsprechung bezüglich der GASP bestehe aber weiterhin ebenso wenig (Art. III-376 VVE) wie ein Interessenten- oder Konkurrentenrechtschutz. Insgesamt gewähre der Verfassungsvertrag partiell mehr Individualschutz als die aktuelle Rechtslage. Allerdings bestünde die Möglichkeit, dass die europäischen Gerichte dieser vorsichtigen Ausweitung durch eine einschränkende Auslegung restriktiv gegenübertreten könnten.

\section{Die Gemeinschaft und ihre Mitgliedstaaten in der Welthandelsorganisation}

Christian Tietje untersuchte, wie sich die weitgreifende Kompetenzübertragung auf die Gemeinschaft im Bereich der Gemeinsamen Handelspolitik auf die parallele Mitgliedschaft der Gemeinschaft und ihrer Mitgliedstaaten in der Welthandelsorganisation auswirken werde. Eine Austrittspflicht sei nicht zu erkennen, allerdings sei fraglich, ob den Mitgliedstaaten in Anbetracht der Reichweite der umfassenden Bindung der Gemeinschaft an das Welthandelsrechts ein relevanter materiellrechtlicher Mitgliedschaftsstatus in der WTO verbliebe. In der Praxis der Streitbeilegungsverfahren der Welthandelsorganisation sei inzwischen die alleinige, vollumfängliche Inanspruchnahme der Gemeinschaft auch für Maßnahmen ihrer Mitgliedstaaten fast ausnahmslose Regel, welche sich bald auch explizit auf die Verantwortlichkeit für den Verwaltungsvollzug und die
Verwaltungsorganisation der Mitgliedstaaten erstrecken könnte (laufendes Verfahren ,EGZollverwaltung'). Inwieweit sich die Gemeinschaft - jedenfalls soweit ihr im Inneren keine Harmonisierungskompetenz zustehe - auf eine Rechtfertigungsmöglichkeit nach Art. XX Buchstabe d) des Allgemeinen Zoll- und Handelsabkommens berufen könne, dürfe im Rahmen des Verfahrens ,Mexico - Tax Measures on Soft Drinks and other Beverages" zum NAFTA-Recht einer baldigen Präzedenzentscheidung zugeführt sein. Bezüglich der Verantwortungsstruktur, nicht aber in Hinblick auf die Führung der laufenden Verhandlungen, bestünde somit bereits heute eine weitgehende Bedeutungslosigkeit der ,mediatisierten“ Mitgliedstaaten, so dass von einem föderalen Konzept im Außenhandelssektor gesprochen werden könne. Dieses lasse insofern eine parallele Mitgliedschaft als obsolet erscheinen.

Wolfgang Weiß analysierte die Auswirkungen des Verfassungsvertrages auf die Rezeption des Welthandelsrechts durch das Gemeinschaftsrecht. Eine unmittelbare Wirkung des Welthandelsrechts sei vom Europäischen Gerichtshof in ständiger Rechtsprechung abgelehnt worden. Lediglich eine Pflicht zur welthandelsrechtskonformen Auslegung des Gemeinschaftsrechts bestünde, welche auch den Mitgliedstaaten bei der Umsetzung von Gemeinschaftsrecht obliege. Eine Schadensersatzpflicht der Gemeinschaft habe der Europäische Gerichtshof im Fall ,Biret' bei einem Verstoß gegen das Welthandelsrecht unter bestimmten Voraussetzungen als Lösungsoption erachtet, allerdings deute die jüngste Rechtsprechung beispielsweise im Fall, Van Parys" auf eine weiterhin restriktive Haltung hin. Der Verfassungsvertrag brächte nach Weiß insofern nun keine Zeitenwende, da die maßgeblichen Normen inhaltlich nahezu unverändert geblieben seien. Beachtlich sei zwar die Verschiebung des institutionellen Gleichgewichts in der Handelspolitik zugunsten des Parlaments, so dass die Aussagen des Gutachtens 1/94 des Europäischen Gerichtshofs insofern an Aussagekraft verlören. Allerdings werde 
sich der Gerichtshof nun wohl erst Recht nicht mehr mit einer eigenmächtigen Anerkennung der unmittelbaren Wirkung über das so gestärkte Parlament hinwegsetzen. Somit bestehe auch nach dem Verfassungsvertrag lediglich eine Pflicht zur welthandelsrechtskonformen Auslegung - mehr als eine Neuakzentuierung der Diskussion lasse sich ihm nicht entnehmen.

Weitere Aspekte der Integration im Außenhandelssektor

Im Rahmen des abschließenden Panels unter der Leitung von Horst G. Krenzler zum Thema ,Harnessing Globalisation“ ging zunächst Meinhard Hilf auf den Konstitutionalisierungsprozess der Welthandelsorganisation ein und bewertete diesen anhand der Maßstäbe des Art. 23 GG. Hierbei verzeichnete er ein Demokratiedefizit, welchem er insbesondere durch Stärkung parlamentarischer Elemente im Sinne eines Beratungs- und Kommunikationsorgans begegnet sehen wollte. Eine Entscheidungsfunktion dieses Gremiums halte er indes nicht für wünschenswert. KarlErnst Brauner ließ eine persönliche Bewertung der Aussichten der ,Doha Development Agenda' folgen. Zwar seien die Verhandlungen von vielen Rückschlägen und massiven Interessenkonflikten geprägt, was sich für die Gemeinschaft nicht zuletzt durch die Ausklammerung fast aller Singapur-Themen manifestiert habe. Dem hierdurch verstärkten Druck zum Abschluss bilateraler Verträge dürfe man im Interesse eines Erfolges der Handelsrunde grundsätzlich nicht nachgeben. Trotz aller Unwägbarkeiten sehe er jüngst Anzeichen für einen erfolgreichen Abschluss der Gespräche. Insbesondere im Agrarsektor und im Bereich des ,special and differential treatment" sei Bewegung in die Diskussion gekommen. Marc Beise richtete das Augen- merk auf die Öffentlichkeit als relevante Begleiterin der Globalisierung. Insofern dürfe man die Welthandelsorganisation als Erfolgsgeschichte betrachten, da es ihr gelungen sei, Globalisierungskritik auch durch erhöhte Transparenz in die Diskussionen einzubeziehen. Hier sei die Welthandelsorganisation vergleichsweise weiter als die Gemeinschaft im Bereich der Handelspolitik. Vor einem verstärkten ,Nutzbarmachen' der Globalisierung müssten nun zunächst die Menschen für den Status quo gewonnen werden. Auf den neuen Trend der strategischen Koalitionen zwischen Nichtregierungsorganisationen und Entwicklungsländern und seine Implikationen für die Arbeit der Welthandelsorganisation wurde hingewiesen. Till Müller-Ibold begrüßte nochmals den Verfassungsentwurf und sah mit ihm die Gemeinschaft bezüglich ihrer großen außenhandelspolitischen Herausforderungen besser gewappnet. Die Kompetenzklärungen seien erforderlich, die bestehenden Ausnahmen zum Prinzip der Gemeinschaftszuständigkeit im Bereich der Gemeinsamen Handelspolitik überdenkenswert.

\section{Fazit}

Die Münchener Tagung bot einen intensiven Einblick in den Regelungskomplex der AuBenwirtschaftspolitik, wobei die Außenhandelspolitik als vorrangiger Betrachtungsgegenstand herangezogen wurde. Der Verfassungsvertrag lässt sich diesbezüglich als behutsame Fortentwicklung der europäischen Integrationstradition samt einer weiteren Kompetenzübertragung auf die Gemeinschaft kennzeichnen. Welcher Kreativität der Wunsch nach Einbeziehung dieser Regelungsleistung in das geltende Recht gegebenenfalls abseits eines Ratifizierungsprozesses begegnen wird, darf mit Spannung erwartet werden. 